\title{
A POISSON SUMMATION FORMULA FOR INTEGRALS OVER QUADRATIC SURFACES
}

\author{
BY
}

ROBERT S. STRICHARTZ ${ }^{1}$

ABSTRACT. Let $S(t)$ denote Lebesgue measure on the sphere of radius $t>0$ in $\mathbf{R}^{n}$, and

$$
S_{k}(t)=\left(\frac{\partial}{\partial t} \frac{1}{t}\right)^{k} S(t) .
$$

Let $P \Sigma_{k}=S_{k}(0)+2 \sum_{m=1}^{\infty} S_{k}(m)$. THEOREM. If $n$ is odd and $j$ and $k$ are nonnegative integers with $j+k=(n-1) / 2$, then the Fourier transform of $P \Sigma_{j}$ is $(2 \pi)^{j-k} P \Sigma_{k}$.

There is an analogous, although slightly different, identity involving integrals over hyperboloids in odd dimensions. These results were inspired by recent work of M. Vergne.

1. Introduction. The Poisson summation formula is the identity $\Sigma_{-\infty}^{\infty} f(m)=$ $\Sigma_{-\infty}^{\infty} \hat{f}(m)$ where $\hat{f}(y)=\int_{-\infty}^{\infty} e^{2 \pi i x y} f(x) d x$ for $f \in \delta\left(\mathbf{R}^{1}\right)$, which is equivalent to the statement that the tempered distribution $\Sigma_{-\infty}^{\infty} \delta(x-m)$ is equal to its own Fourier transform. Quite recently, Michele Vergne [4] has discovered some striking generalizations of this identity, motivated by the theory of representations of semisimple Lie groups. For example, in connection with the rotation group $S O(3)$, she proves

$$
\varphi(0)+2 \sum_{1}^{\infty} \frac{1}{4 \pi m^{2}}\langle S(m), \varphi\rangle=\sum_{1}^{\infty}\left\langle S\left(m-\frac{1}{2}\right),\left(\frac{\sin \pi|x|}{\pi|x|} \varphi\right)\right\rangle
$$

for all $\varphi \in \delta\left(\mathbf{R}^{3}\right)$ where $S(r)$ denotes the Lebesgue measure on the sphere of radius $r$ in $\mathbf{R}^{3}$.

Inspired by this result, we have found a number of identities in which a distribution supported on a union of lower-dimensional submanifolds of $\mathbf{R}^{n}$ has a Fourier transform with similar support. We propose calling any such identity a Poisson summation formula. While we do not have any particular applications in mind, we believe that the simplicity and elegance of these identities makes them worthy of attention.

The basic method for deriving such identities is extremely simple. First note that the original Poisson summation formula applied to even functions is equivalent to the identity

$$
\int_{0}^{\infty} f(r) d r+2 \sum_{1}^{\infty} \int_{0}^{\infty} f(r) \cos 2 \pi m r d r=\frac{1}{2} f(0)+\sum_{1}^{\infty} f(m) .
$$

Received by the editors November 24, 1980.

1980 Mathematics Subject Classification. Primary 42B10.

Key words and phrases. Poisson summation formula.

'Research supported in part by NSF grant MCS-8002771.

(C) 1982 American Mathematical Society 0002-9947/81/0000-1028/\$03.75 
It is not necessary for $f$ to belong to $\delta$ for this to hold; it suffices for $f$ and $f^{\prime}$ to be continuous and rapidly decreasing for example, and we shall need this in the proof of Theorem 2 below. Now if $T(t)$ and $S(t)$ are two families of distributions in $\mathbf{R}^{n}$ that are related by an identity

$$
T(t) \hat{)}=c \int_{0}^{\infty} S(r) \cos 2 \pi r t d r,
$$

then we may substitute $f(r)=\langle S(r), \varphi\rangle$ for $\varphi \in \varsigma\left(\mathbf{R}^{n}\right)$ into (1.2) to obtain

$$
\left(S(0)+2 \sum_{1}^{\infty} S(m)\right) \hat{=} c^{\prime}\left(T(0)+2 \sum_{1}^{\infty} T(m)\right)
$$

where $c^{\prime}=2 / c$. Thus, modulo technical details, any identity of the form (1.3), where $T(t)$ and $S(t)$ have support on lower-dimensional submanifolds, automatically leads to a Poisson summation formula of the form (1.4). We also note that the variant of (1.2),

$$
\int_{0}^{\infty} f(r) d r+2 \sum_{1}^{\infty}(-1)^{m} \int_{0}^{\infty} f(r) \cos 2 \pi m r d r=\sum_{1}^{\infty} f\left(m-\frac{1}{2}\right),
$$

which is obtained by applying the original Poisson summation formula to $f\left(x-\frac{1}{2}\right)$, leads to the variant

$$
\left.\left(2 \sum_{1}^{\infty} S\left(m-\frac{1}{2}\right)\right)\right)^{\prime}\left(T(0)+2 \sum_{1}^{\infty}(-1)^{m} T(m)\right) .
$$

It turns out that relations of the form (1.3) are fairly common in odd-dimensional spaces. We give some examples involving distributions supported on spheres in $\$ 2$ and on hyperboloids in \$3. Undoubtedly more examples await discovery. Our identities overlap with Vergne's only in the case of $S O(3)$ cited above.

We are grateful to Michele Vergne for bringing her work to our attention and suggesting this line of investigation.

2. Integrals over spheres. Let $n$ be an odd integer, $n \neq 1$ to avoid triviality. For $\varphi \in \delta\left(\mathbf{R}^{n}\right)$ let $\hat{\varphi}(y)=\int_{\mathbf{R}^{n}} e^{2 \pi i x y} \varphi(x) d x$. Define $S(r)$ to be Lebesgue measure on the sphere of radius $r$; thus we have the polar coordinates formula

$$
\int_{\mathbf{R}^{n}} \varphi(x) d x=\int_{0}^{\infty}\langle S(r), \varphi\rangle d r .
$$

We also define $S_{k}(t)=\left(\frac{\partial}{\partial t} \frac{1}{t}\right)^{k} S(t)$, a distribution supported on the sphere of radius $t$. For $k \leqslant(n-1) / 2$ we may also take $S_{k}(0)=\lim _{t \rightarrow 0} S_{k}(t)$. In fact it is easy to show $S_{k}(0)=0$ for $0 \leqslant k \leqslant(n-3) / 2$, while $S_{(n-1) / 2}(0)=2(2 \pi)^{(n-1) / 2} \delta$. Now it is well known that the Fourier transforms of $S_{k}(t)$ are expressible in terms of Bessel functions, and it is not difficult to cast this in the form (1.3).

LemMa 1. Let $j, k$ be nonnegative integers with $j+k=(n-1) / 2$. Then

$$
\left\langle S_{k}(t), \hat{\varphi}\right\rangle=2(2 \pi)^{k-j} \int_{0}^{\infty}\left\langle S_{j}(r), \varphi\right\rangle \cos 2 \pi t r d r
$$

for any $\varphi \in \S\left(\mathbf{R}^{n}\right)$. 
Proof. We begin with the well-known Fourier transform formula

$$
\langle S(t), \hat{\varphi}\rangle=2 \pi t^{n / 2} \int|x|^{1-n / 2} J_{n / 2-1}(2 \pi t|x|) \varphi(x) d x
$$

(see [2, p. 154]) from which we obtain

$$
\begin{aligned}
\left\langle S_{k}(t), \hat{\varphi}\right\rangle & =(2 \pi)^{k+1} t^{n / 2-k} \int|x|^{k+1-n / 2} J_{n / 2-1-k}(2 \pi t|x|) \varphi(x) d x \\
& =(2 \pi)^{k+1} t^{n / 2-k} \int_{0}^{\infty} r^{k+1-n / 2} J_{n / 2-1-k}(2 \pi t r)\langle S(r), \varphi\rangle d r
\end{aligned}
$$

using the recursion relation for the Bessel functions $\frac{d}{d t}\left(t^{\alpha} J_{\alpha}(t)\right)=t^{\alpha} J_{\alpha-1}(t)$ and the polar coordinates formula. Notice that this is (2.2) when $k=(n-1) / 2$ since $J_{-1 / 2}(t)=\sqrt{\frac{2}{\pi}} t^{-1 / 2} \cos t$. For the general case we integrate by parts $j$ times on the right in (2.3), using the recursion relation $\frac{d}{d t}\left(t^{-\alpha} J_{\alpha}(t)\right)=-t^{-\alpha} J_{\alpha+1}(t)$, and note that no boundary terms arise because $t^{-\alpha} J_{\alpha}(t)$ is bounded as $t \rightarrow 0$.

THEOREM 1. Let $P \Sigma_{k}=S_{k}(0)+2 \sum_{m=1}^{\infty} S_{k}(m)$. Then

$$
\left(P \Sigma_{j}\right)^{\hat{n}}=(2 \pi)^{j-k} P \Sigma_{k}
$$

if $j+k=(n-1) / 2, j, k$ nonnegative integers. More explicitly, if $\varphi \in S\left(\mathbf{R}^{n}\right)$ then

$$
\sum_{1}^{\infty}\langle S(m), \varphi\rangle=\hat{\varphi}(0)+\sum_{1}^{\infty}(2 \pi)^{(1-n) / 2}\left\langle S_{(n-1) / 2}(m), \hat{\varphi}\right\rangle
$$

while if $1 \leqslant j \leqslant(n-3) / 2$ then

$$
\sum_{1}^{\infty}\left\langle S_{j}(m), \varphi\right\rangle=(2 \pi)^{j-k} \sum_{1}^{\infty}\left\langle S_{k}(m), \hat{\varphi}\right\rangle \text {. }
$$

In particular, if $n \equiv 1 \bmod 4$ then

$$
\sum_{1}^{\infty}\left\langle S_{(n-1) / 4}(m), \varphi\right\rangle=\sum_{1}^{\infty}\left\langle S_{(n-1) / 4}(m), \hat{\varphi}\right\rangle \text {. }
$$

We also have

$$
\sum_{1}^{\infty}\left\langle S\left(m-\frac{1}{2}\right), \hat{\varphi}\right\rangle=\varphi(0)+\sum_{1}^{\infty}(2 \pi)^{(1-n) / 2}(-1)^{m}\left\langle S_{(n-1) / 2}(m), \hat{\varphi}\right\rangle .
$$

Proof. Since $\left(1 / r^{n-1}\right)\langle S(r), \varphi\rangle$ is $C^{\infty}$ at $r=0$ and may be extended to be even in $r$, since $n$ is odd, it follows that for $j \leqslant(n-1) / 2,\left\langle S_{j}(r), \varphi\right\rangle$ extended to be even in $r$ is in $\delta\left(\mathbf{R}^{1}\right)$, so that we may substitute $f(r)=\left\langle S_{j}(r), \varphi\right\rangle$ in (1.2). The identity that results from Lemma 1 is then (2.4). To obtain (2.8) we use (1.2') and Lemma 1.

COROLlaRy 1. If $\varphi \in \varsigma\left(\mathbf{R}^{n}\right)$ and $\varphi$ has support in the unit ball then

$$
\varphi(0)=\sum_{1}^{\infty}\langle S(m), \hat{\varphi}\rangle=\sum_{1}^{\infty}\left\langle S\left(m-\frac{1}{2}\right), \hat{\varphi}\right\rangle \text {. }
$$


If the support of $\varphi$ is contained in $|x| \leqslant \frac{1}{2}$ then

(2.10) $\varphi(x)=\sum_{1}^{\infty}\left\langle S(m), e^{-2 \pi i(x \cdot \xi)} \hat{\varphi}(\xi)\right\rangle=\sum_{1}^{\infty}\left\langle S\left(m-\frac{1}{2}\right), e^{-2 \pi i(x \cdot \xi)} \hat{\varphi}(\xi)\right\rangle$ for all $|x| \leqslant \frac{1}{2}$.

Proof. These local Fourier inversion formulas are obtained from (2.5) with $\hat{\varphi}$ in place of $\varphi$, since all but the first term on the right vanish. We obtain $(2.10)$ by applying (2.9) to a translate of $\varphi$.

Corollary 2. Let $g(x)=(\sin (\pi|x|) / \pi|x|)^{(n-1) / 2}$. Then

$$
\begin{gathered}
\varphi(0)+\sum_{1}^{\infty}(-1)^{m(n-1) / 2}(2 \pi)^{(1-n) / 2}\left(\frac{n-1}{2}\right) ! m^{1-n}\langle S(m), \varphi\rangle \\
=\sum_{1}^{\infty}\left\langle S(m),(g \varphi)^{\wedge}\right\rangle
\end{gathered}
$$

and

$$
\begin{gathered}
\varphi(0)+\sum_{1}^{\infty}(-1)^{m(n+1) / 2}(2 \pi)^{(1-n) / 2}\left(\frac{n-1}{2}\right) ! m^{1-n}\langle S(m), \varphi\rangle \\
=\sum_{1}^{\infty}\left\langle S\left(m-\frac{1}{2}\right),(g \varphi)^{\wedge}\right\rangle .
\end{gathered}
$$

Proof. If we substitute $(g \varphi) \hat{\text { for }} \varphi$ in (2.5) we obtain

$$
\sum_{1}^{\infty}\left\langle S(m),(g \varphi)^{\wedge}\right\rangle=g(0) \varphi(0)+\sum_{1}^{\infty}(2 \pi)^{(1-n) / 2}\left\langle S_{(n-1) / 2}(m), g \varphi\right\rangle .
$$

Now,

$$
\left\langle S_{(n-1) / 2}(m), g \varphi\right\rangle=\left.\left(\frac{d}{d t} \frac{1}{t}\right)^{(n-1) / 2}\left(\frac{\sin \pi t}{\pi t}\right)^{(n-1) / 2}\langle S(t), \varphi\rangle\right|_{t=m} .
$$

Since each factor $\sin \pi t$ vanishes at $t=m$ we will only get a nonzero contribution if each derivative is applied to a sin $\pi t$ factor. Thus

$$
\begin{aligned}
\left.\left(\frac{\partial}{\partial t} \frac{1}{t}\right)^{(n-1) / 2}\left(\frac{\sin \pi t}{\pi t}\right)^{(n-1) / 2}\langle S(t), \varphi\rangle\right|_{t=m} & \\
= & \left.\left(\frac{n-1}{2}\right) ! t^{1-n}(\cos \pi t)^{(n-1) / 2}\langle S(t), \varphi\rangle\right|_{t=m} \\
& =\left(\frac{n-1}{2}\right) !(-1)^{m(n-1) / 2} m^{1-n}\langle S(m), \varphi\rangle
\end{aligned}
$$

so we obtain (2.11). Similarly (2.12) follows from (2.8).

Note that (2.12) for $n=3$ is the identity (1.1) of Vergne.

3. Integrals over hyperboloids. Let $Q$ be the nondegenerate quadratic form

$$
Q(x, x)=\sum_{i=1}^{p} x_{i}^{2}-\sum_{i=p+1}^{n} x_{i}^{2}
$$


on $\mathbf{R}^{n}$, and set $q=n-p$. We will assume $n$ is odd, and to be specific take $p$ even and $q$ odd, $p \neq 0$, so we are in the indefinite case. The level sets of $Q$ form two families of hyperboloids, $Q=r^{2}$ and $Q=-r^{2}$, and the cone $Q=0$. Each of these sets carries a natural measure which can be described simply in local coordinates as $\left(d x_{1} \ldots \widehat{d x}_{j} \ldots d x_{n}\right) /\left|x_{j}\right|$ in a neighborhood where $x_{j} \neq 0$, and $x_{j}$ is a function of the other variables obtained by solving the equation $Q(x, x)=$ constant. We denote this measure by $H^{+}(r)$ for the $Q(x, x)=r^{2}$ hyperboloids, $H^{-}(r)$ for the $Q(x, x)=$ $-r^{2}$ hyperboloids, and $H(0)$ for the $Q(x, x)=0$ cone. Note that these are infinite positive measures and as distributions are tempered. They can be uniquely characterized, up to a constant multiple, in any one of three ways: (1) as the limit in the distribution sense of $\varphi_{\varepsilon}(Q(x, x))$ where $\varphi_{\varepsilon} \in \mathcal{D}\left(\mathbf{R}^{1}\right)$ approximates the delta function at $\pm r^{2}$; (2) as the invariant measure under the transitive action of the pseudo-orthogonal group $O(p, q)$; (3) as the measure determined by the possibly indefinite Riemannian metric inherited from the indefinite Riemannian metric $Q(x, x)$ on the ambient space $\mathbf{R}^{n}$. The reader should be careful to note that this is a different measure than the usual surface integral which is determined from the definite Riemannian metric inherited from the $x \cdot x$ Euclidean metric on $\mathbf{R}^{n}$.

We note that with the normalization we have chosen, $H(0)$ is the limit of $H^{ \pm}(r)$ as $r \rightarrow 0$. Also we have the "polar" coordinates formula

$$
\int_{Q(x, x)>0} \varphi(x) d x=\int_{0}^{\infty}\left\langle H^{+}(r), \varphi\right\rangle r d r .
$$

Note that this is not the exact analogue of (2.1). We define $H_{k}^{+}(r)=\left(\frac{1}{r} \frac{\partial}{\partial r}\right)^{k} H^{+}(r)$. Again this differs from the definition of $S_{k}(r)$.

Lemma 2. Let $k \leqslant(n-3) / 2$ and $\varphi \in \mathcal{S}\left(\mathbf{R}^{n}\right)$. Then $\left\langle H_{k}^{+}(r), \varphi\right\rangle$, extended to be an even function in $r$, is $C^{1}$ and, together with its derivative, rapidly decreasing. In particular, $\left\langle H_{k}(0), \varphi\right\rangle=\lim _{r \rightarrow 0}\left\langle H_{k}^{+}(r), \varphi\right\rangle$ exists and defines a distribution supported on the cone.

Proof. These facts are most easily seen by expressing $H(r)$ in terms of bipolar coordinates. Let $u \in S^{p-1}$ and $v \in S^{q-1}, s>0, t>0$ coordinatize the point $x=$ $(s u, t v)$. A simple computation shows

$$
\begin{aligned}
& \left\langle H^{+}(r), \varphi\right\rangle \\
& \quad=\int_{S^{p-1}} \int_{S^{q-1}} \int_{0}^{\infty} \varphi\left(\sqrt{r^{2}+t^{2}} u, t v\right)\left(t^{2}+r^{2}\right)^{(p-2) / 2} t^{q-1} d t d v d u .
\end{aligned}
$$

The expression on the right is already an even function of $r \in \mathbf{R}$, and is clearly $C^{\infty}$ for $r \neq 0$ and rapidly decreasing as $r \rightarrow \infty$. We need to examine the behavior of this function near $r=0$. It is clearly continuous, and

$$
\langle H(0), \varphi\rangle=\int_{S^{p-1}} \int_{S^{q-1}} \int_{0}^{\infty} \varphi(t u, t v) t^{n-3} d t d v d u .
$$


Now we compute

$$
\begin{aligned}
& \frac{1}{r} \frac{\partial}{\partial r}\left\langle H^{+}(r), \varphi\right\rangle \\
& \quad=\int_{S^{p-1}} \int_{S^{q-1}} \int_{0}^{\infty}\left((p-2) \varphi\left(\sqrt{r^{2}+t^{2}} u, t v\right)\left(t^{2}+r^{2}\right)^{(p-4) / 2} t^{q-1}\right. \\
& \left.\quad+(u \cdot \nabla) \varphi\left(\sqrt{r^{2}+t^{2}} u, t v\right)\left(t^{2}+r^{2}\right)^{(p-3) / 2} t^{q-1}\right) d t d v d u .
\end{aligned}
$$

Hence by induction

$$
\begin{array}{r}
\left\langle H_{k}^{+}(r), \varphi\right\rangle=\sum_{j=0}^{k} \int_{S^{p-1}} \int_{S^{q-1}} \int_{0}^{\infty} c_{j}(u \cdot \nabla)^{j} \varphi\left(\sqrt{r^{2}+t^{2}} u, t v\right) \\
\cdot\left(t^{2}+r^{2}\right)^{(p-2-2 k+j) / 2} t^{q-1} d t d v d u
\end{array}
$$

for certain constants $c_{j}$. As long as $k \leqslant(n-3) / 2$ we can take the limit as $r \rightarrow 0$ to get

$$
\left\langle H_{k}(0), \varphi\right\rangle=\sum_{j=0}^{k} \int_{S^{p-1}} \int_{S^{q-1}} \int_{0}^{\infty} c_{j}(u \cdot \nabla)^{j} \varphi(t u, t v) t^{(n-3-2 k+j) / 2} d t d v d u
$$

since the power of $t$ is nonnegative. Finally note that differentiating $\frac{\partial}{\partial r}\left\langle H_{k}^{+}(r), \varphi\right\rangle$ will produce additional factors of the form $r / \sqrt{r^{2}+t^{2}}$ which are bounded uniformly, so $\lim _{r \rightarrow 0} \frac{\partial}{\partial r}\left\langle H_{k}^{+}(r), \varphi\right\rangle=0$ by the dominated convergence theorem.

Lemma 3. Let $j, k$ be nonnegative integers such that $j+k=(n-3) / 2$. Then

$$
\left\langle H_{k}^{+}(t), \hat{\varphi}\right\rangle=(-1)^{(q-1) / 2} 2(2 \pi)^{k-j} \int_{0}^{\infty}\left\langle H_{j}^{+}(r), \varphi\right\rangle \cos 2 \pi r t d r
$$

for all $\varphi \in \varsigma\left(\mathbf{R}^{n}\right)$, and $t \geqslant 0$.

Proof. The computation of Fourier transforms of distributions which are functions of $Q(x, x)$ has been carried out by the author in [3] based on ideas from Gelfand and Shilov [1]. From Theorem 1 of [3] we have the identity

$$
\begin{aligned}
& \langle f(Q(x, x)), \hat{\varphi}\rangle \\
& \quad=-2 \pi \sin \frac{\pi}{2} q \int_{0}^{\infty}\left\langle Y_{(n-2) / 2}\left(2 \pi t Q(x, x)^{1 / 2}\right) Q(x, x)_{+}^{(2-n) / 4}, \varphi\right\rangle f(t) t^{n / 2} d t
\end{aligned}
$$

for $f \in S\left(\mathbf{R}^{l}\right)$ with support in $t \geqslant 0$ and $\varphi \in S\left(\mathbf{R}^{n}\right)$. Here we use the notation

$$
Q(x, x)_{+}^{\lambda}= \begin{cases}Q(x, x)^{\lambda} & \text { if } Q(x, x)>0 \\ 0 & \text { if } Q(x, x)<0\end{cases}
$$

and $Y$ denotes the Neumann function ( $N$ in Russian translations such as [1]). We have adjusted this identity by appropriate factors of $2 \pi$ to take into account the different definition of the Fourier transform in [3], namely, $\int e^{i Q(x, y)} \varphi(x) d x$ (the use of $Q(x, y)$ in place of $x \cdot y$ does not result in any change because $Q(x, y)$ is even in each variable). 
Since the index $(n-2) / 2$ is half-integral for $n$ odd, we can replace the Neumann function by a Bessel function, $Y_{(n-2) / 2}=(-1)^{(n-1) / 2} J_{(2-n) / 2}$. By taking $f$ to approximate the delta function at $t$ and comparing with (3.1) we obtain

$$
\begin{aligned}
& \left\langle H^{+}(t), \hat{\varphi}\right\rangle \\
& \quad=-2 \pi(-1)^{p / 2} t^{(n-2) / 2}\left\langle J_{(2-n) / 2}\left(2 \pi t Q(x, x)^{1 / 2}\right) Q(x, x)_{+}^{(2-n) / 4}, \varphi\right\rangle .
\end{aligned}
$$

Unfortunately, except for the case when $n=3$, the distribution

$$
J_{(2-n) / 2}\left(2 \pi t Q(x, x)^{1 / 2}\right) Q(x, x)_{+}^{(2-n) / 4}
$$

is not given by a locally integrable function, and must be defined by a process of regularization or analytic continuation. Were it not for this fact, we could easily complete the proof by writing

$$
\left\langle H^{+}(t), \hat{\varphi}\right\rangle=-2 \pi(-1)^{p / 2} t^{(n-2) / 2} \int_{0}^{\infty}\left\langle H^{+}(r), \varphi\right\rangle J_{(2-n) / 2}(2 \pi r t) r^{(4-n) / 2} d r,
$$

differentiating to obtain

$$
\begin{aligned}
\left\langle H_{k}^{+}(t), \hat{\varphi}\right\rangle= & (-1)^{p / 2+k+1}(2 \pi)^{k+1} t^{(n-2) / 2+k} \\
& \cdot \int_{0}^{\infty}\left\langle H^{+}(r), \varphi\right\rangle J_{k+(2-n) / 2}(2 \pi r t) r^{k+2-n / 2} d r
\end{aligned}
$$

and then integrating by parts $j$ times to obtain (3.3). This argument is in fact perfectly valid as long as $\varphi$ vanishes in a neighborhood of the cone; but this is not a restriction we wish to make, so we must take a more roundabout route.

An examination of the proof of Theorem 1 in [3] reveals that (3.5) is really an abbreviation for the identity

$$
\begin{aligned}
\left\langle H^{+}(t), \hat{\varphi}\right\rangle= & -2 \pi(-1)^{p / 2} \sum_{\mu=\nu}^{\infty} \frac{(-1)^{\nu+\mu} t^{2 \mu-2 \nu}}{\mu ! \Gamma(\mu+2-n / 2)} \pi^{(2-n) / 2+2 \mu} \\
& \cdot\left\langle Q(x, x)_{+}^{(2-n) / 2+\mu}, \square^{\nu} \varphi\right\rangle
\end{aligned}
$$

where $\square=Q\left(\frac{\partial}{\partial x}, \frac{\partial}{\partial x}\right)$ and $\nu$ is chosen large enough that all the functions $Q(x, x)_{+}^{(2-n) / 2+\mu}$ are locally integrable $(\nu=(n-1) / 2$ will do). We also note the identity (equation (2.2) of [3])

$$
\square^{\nu} Q(x, x)_{+}^{\lambda}=a(\nu, \lambda) Q(x, x)_{+}^{\lambda-\nu}
$$

for all $\lambda>\nu-1$ where $a(\nu, \lambda)^{-1}=2^{\nu}(\lambda+1) \cdots(\lambda+\nu)(n+2 \lambda) \cdots(n+2 \lambda+$ $2 \nu-2)$. If we were to formally integrate by parts in $\left(3.5^{\prime}\right)$ using (3.6) then we would obtain (3.5) in series form; but the restriction $\lambda>\nu-1$ for (3.6) to hold prohibits doing this for all terms unless we extend the definition of $Q(x, x)_{+}^{\lambda}$ as a distribution by analytic continuation using (3.6).

Next let us differentiate $\left(3.5^{\prime}\right)$, applying $\left(\frac{1}{t} \frac{\partial}{\partial t}\right)^{k}$ to the right side, to obtain

$$
\begin{aligned}
\left\langle H_{k}^{+}(t), \hat{\varphi}\right\rangle= & -2 \pi(-1)^{p / 2} \sum_{\mu=0}^{\infty} \frac{(-1)^{\mu+k}(\mu+1) \cdots(\mu+k)}{(\mu+\nu+k) ! \Gamma(2-n / 2+\mu+\nu+k)} \\
& \cdot 2^{k} \pi^{(2-n) / 2+2 \mu+2 \nu+2 k}\left\langle Q(x, x)_{+}^{(2-n) / 2+\mu+\nu+k}, \square^{\nu} \varphi\right\rangle t^{2 \mu}
\end{aligned}
$$


where we have changed the index of summation. In order to effect the integration by parts on the right side we observe first that if $\operatorname{Re} \lambda>-1$ we have, for $j \leqslant(n-3) / 2$,

$$
\begin{aligned}
\left\langle Q(x, x)_{+}^{\lambda}, \varphi\right\rangle & =\int_{0}^{\infty} r^{2 \lambda+1}\left\langle H^{+}(r), \varphi\right\rangle d r \\
& =\frac{(-1)^{j}}{(2 \lambda+2) \cdots(2 \lambda+2 j)} \int_{0}^{\infty} r^{2 \lambda+2 j+1}\left\langle H_{j}^{+}(r), \varphi\right\rangle d r,
\end{aligned}
$$

the integration by parts being justified by Lemma 2 , and no boundary terms arising since $\operatorname{Re} \lambda>-1$. Thus if $\operatorname{Re} \lambda>\nu-1$ we can write

$$
\left\langle Q(x, x)_{+}^{\lambda}, \square^{\nu} \varphi\right\rangle=a(\nu, \lambda)\left\langle Q(x, x)_{+}^{\lambda-\nu}, \varphi\right\rangle,
$$

hence by (3.8),

$$
\begin{aligned}
\left\langle Q(x, x)_{+}^{\lambda}, \square^{\nu} \varphi\right\rangle= & \frac{(-1)^{j} a(\nu, \lambda)}{(2 \lambda-2 \nu+2) \cdots(2 \lambda-2 \nu+2 j)} \\
& \cdot \int_{0}^{\infty} r^{2 \lambda-2 \nu+2 j+1}\left\langle H_{j}^{+}(r), \varphi\right\rangle d r .
\end{aligned}
$$

But both sides of (3.9) are analytic functions of $\lambda$ in the half-plane $\operatorname{Re} \lambda>\nu-1-j$ except for certain integer values, for $j \leqslant(n-3) / 2$. Thus by analytic continuation (3.9) continues to hold for these values of $\lambda$. In particular, if $j+k=$ $(n-3) / 2$, the value $\lambda=(2-n) / 2+\mu+\nu+k$, for $\mu$ a nonnegative integer, is half-integral and satisfies $\lambda>\nu-1-j$, so we may use (3.9) on the right side of (3.7) to obtain

$$
\left\langle H_{k}^{+}(t), \hat{\varphi}\right\rangle=\sum_{\mu=0}^{\infty} c_{\mu} \int_{0}^{\infty} r^{2 \mu} t^{2 \mu}\left\langle H_{j}^{+}(r), \varphi\right\rangle d r
$$

where, after considerable simplification, we find

$$
c_{\mu}=(-1)^{(q-1) / 2} 2(2 \pi)^{k-j} \frac{(-1)^{\mu}(2 \pi)^{2 \mu}}{(2 \mu) !} .
$$

Thus we have the cosine power series, which we can interchange with the integral to obtain (3.3).

THEOREM 2. Let $P \Sigma_{k}^{+}=H_{k}(0)+2 \sum_{m=1}^{\infty} H_{k}^{+}(m)$. Then

$$
\left(P \Sigma_{k}^{+}\right)^{\hat{n}}=(-1)^{(q-1) / 2}(2 \pi)^{k-j} P \Sigma_{j}^{+}
$$

if $j$ and $k$ are nonnegative integers with $j+k=(n-3) / 2$. In particular, if $n \equiv 3$ $\bmod 4$, then

$$
\left(P \Sigma_{(n-3) / 4}^{+}\right)^{\hat{n}}=(-1)^{(q-1) / 2} P \Sigma_{(n-3) / 4}^{+} .
$$

Proof. Lemma 2 justifies substituting $f(r)=\left\langle H_{k}^{+}(r), \varphi\right\rangle$ in (1.2), and then Lemma 3 gives (3.11). 
In general there do not appear to be analogous identities for the distributions $H^{-}(m)$. However, when $q=1$, there is a substitute result. In that case the hyperboloids $Q(x, x)=-m^{2}$ divide into two connected sheets depending on the sign of $x_{n}$, and the same is true for the cone $Q(x, x)=0$ with the origin deleted. Let us denote by $\operatorname{Sgn} H(t)$ the distribution $\operatorname{sgn} x_{n} H^{-}(t)$, and similarly $\operatorname{Sgn} H(0)=$ $\operatorname{sgn} x_{n} H(0)$. We set $\operatorname{Sgn} H_{k}(t)=\left(\frac{1}{t} \frac{\partial}{\partial t}\right)^{k} \operatorname{Sgn} H(t)$. In terms of bipolar coordinates $\left(S^{q-1}\right.$ is \pm 1$)$

$\langle\operatorname{Sgn} H(r), \varphi\rangle=\int_{S^{p-1}} \int_{0}^{\infty}\left(\varphi\left(t u, \sqrt{r^{2}+t^{2}}\right)-\varphi\left(t u,-\sqrt{r^{2}+t^{2}}\right)\right)$

$$
\cdot\left(r^{2}+t^{2}\right)^{-1 / 2} t^{p-1} d t d u
$$

so the analogue of Lemma 2 holds with essentially the same proof. The methods of [3] are again available for computing the Fourier transforms of Sgn $H_{k}(r)$, because $\operatorname{sgn} x_{n}=\operatorname{sgn} Q(x, \xi)$ in the region $Q(x, x)<0$ (and a.e. on $Q(x, x)=0$ ) for $\xi=(0, \ldots, 0,1,-1)$, and $\operatorname{sgn} Q(x, \xi)$ is a homogeneous wave of degree 0 and odd parity. We also note that since these are odd functions we must adjust the formulas in [3] by a minus sign to account for the $e^{i Q(x, y)}$ in the definition of the Fourier transform used there. From Theorem 1 of [3], with $\sigma=0, \varepsilon=1$, we deduce the analogue of (3.5)

$\langle\operatorname{Sgn} H(t), \hat{\varphi}\rangle$

$$
=2 \pi i t^{(n-2) / 2}\left\langle J_{(2-n) / 2}\left(2 \pi t|Q(x, x)|^{1 / 2}\right) \operatorname{sgn} x_{n} Q(x, x)_{-}^{(2-n) / 4}, \varphi\right\rangle .
$$

From this we obtain by the same reasoning as in Lemma 3, the analogue of (3.3)

$\left\langle\operatorname{Sgn} H_{k}(t), \hat{\varphi}\right\rangle=(-1)^{(n-3) / 2} i 2(2 \pi)^{k-j} \int_{0}^{\infty}\left\langle\operatorname{Sgn} H_{j}(r), \varphi\right\rangle \cos 2 \pi r t d r$.

Thus we obtain

TheOREM 3. Let $P \Sigma_{k}^{-}=\operatorname{Sgn} H_{k}(0)+2 \sum_{m=1}^{\infty} \operatorname{Sgn} H_{k}(m)$. Then

$$
\left(P \Sigma_{k}^{-}\right)^{\hat{n}}=(-1)^{(n-3) / 2} i(2 \pi)^{k-j} P \Sigma_{j}^{-}
$$

if $j$ and $k$ are nonnegative integers with $j+k=(n-3) / 2$. In particular, if $n \equiv 3$ $\bmod 4$, then

$$
\left(P \Sigma_{(n-3) / 4}^{-}\right)^{\wedge}=(-1)^{(n-3) / 2} i P \Sigma_{(n-3) / 4}^{-} .
$$

Finally, if $n$ is even, while there do not appear to be any analogous identities involving integrals over hyperboloids, there are simpler identities involving the cone.

THEOREM 4. If $p$ and $q$ are even, and $j, k$ are nonnegative integers such that $j+k=(n-4) / 2$, then

$$
H_{k}(0)^{\hat{}}=(-1)^{q / 2}(2 \pi)^{k-j} H_{j}(0) .
$$

If $p$ is odd and $q=1$, with $j+k=(n-4) / 2$ as before, then

$$
\operatorname{Sgn} H_{k}(0)^{\hat{n}}=(-1)^{(p-1) / 2} i(2 \pi)^{k-j} \operatorname{Sgn} H_{j}(0) .
$$


Proof. We consider the expression $\left\langle Q(x, x)_{+}^{\lambda}, \varphi\right\rangle$ for $\varphi \in S\left(\mathbf{R}^{n}\right)$ as an analytic function in $\lambda$ in the half-space $\operatorname{Re} \lambda>-1$. We wish to show that it admits a meromorphic continuation into $\operatorname{Re} \lambda>\frac{-n}{2}$, and

$$
\left\langle H_{j}(0), \varphi\right\rangle=2^{j+1} j ! \operatorname{Res}_{\lambda=-j-1}\left\langle Q(x, x)_{+}^{\lambda}, \varphi\right\rangle
$$

for $j \leqslant(n-4) / 2$. (See Gelfand and Shilov [1] for the meromorphic continuation to the entire complex plane and a different computation of the residues.) Indeed we have

$$
\left\langle Q(x, x)_{+}^{\lambda}, \varphi\right\rangle=\int_{0}^{\infty}\left\langle H^{+}(r), \varphi\right\rangle r^{2 \lambda+1} d r
$$

for $\operatorname{Re} \lambda>-1$, hence by integration by parts

$$
\begin{aligned}
& \left\langle Q(x, x)_{+}^{\lambda}, \varphi\right\rangle \\
& =\left(-\frac{1}{2}\right)^{j} \frac{1}{(\lambda+1) \cdots(\lambda+j)} \int_{0}^{\infty}\left\langle H_{j}^{+}(r), \varphi\right\rangle r^{2 \lambda+2 j+1} d r,
\end{aligned}
$$

the integral being convergent for $j \leqslant(n-4) / 2$ by the proof of Lemma 2 . But the integral on the right side is analytic in $\operatorname{Re} \lambda>-j-1$ and can be meromorphically continued to $\operatorname{Re} \lambda>-j-2$ by

$$
\begin{aligned}
\int_{0}^{\infty}\left\langle H_{j}^{+}(r), \varphi\right\rangle r^{2 \lambda+2 j+1} d r= & \int_{1}^{\infty}\left\langle H_{j}^{+}(r), \varphi\right\rangle r^{2 \lambda+2 j+1} d r \\
& +\int_{0}^{1}\left(\left\langle H_{j}^{+}(r), \varphi\right\rangle-\left\langle H_{j}(0), \varphi\right\rangle\right) r^{2 \lambda+2 j+1} d r \\
& +\frac{1}{2(\lambda+j+1)}\left\langle H_{j}(0), \varphi\right\rangle
\end{aligned}
$$

from which we read off

$$
\underset{\lambda=-j-1}{\operatorname{Res}} \int_{0}^{\infty}\left\langle H_{j}^{+}(r), \varphi\right\rangle r^{2 \lambda+2 j+1} d r=\frac{1}{2}\left\langle H_{j}(0), \varphi\right\rangle .
$$

Substituting this into the right side of (3.16) we obtain (3.15).

However, for $p$ even, we have

$$
\begin{aligned}
\left\langle Q(x, x)_{+}^{\lambda}, \hat{\varphi}\right\rangle= & -\pi^{-2 \lambda-1-n / 2} \Gamma(\lambda+1) \Gamma\left(\lambda+\frac{n}{2}\right) \\
& \cdot \sin \pi\left(\lambda+\frac{q}{2}\right)\left\langle Q(x, x)_{+}^{-\lambda-n / 2}, \varphi\right\rangle
\end{aligned}
$$

(see [3, Lemma 2]). Let us take the residue at $\lambda=-k-1$ of both sides of this identity between meromorphic functions. Note that $-\lambda-\frac{n}{2}=-j-1$, and the product $\Gamma(\lambda+1) \sin \pi\left(\lambda+\frac{q}{2}\right)$ is regular at $\lambda=-k-1$ with value $(-1)^{q / 2+1} \pi / k$ ! since $q$ is even, hence we obtain

$$
\frac{1}{2^{k+1} k !}\left\langle H_{k}(0), \hat{\varphi}\right\rangle=-\pi^{2 k+1-n / 2} \Gamma\left(\frac{n}{2}-k-1\right) \frac{(-1)^{q / 2+1} \pi}{k !} \frac{1}{2^{j+1} j !}\left\langle H_{j}(0), \varphi\right\rangle
$$

which simplifies to (3.13). 
The proof of (3.14) is similar; in place of (3.15) we have

$$
\left\langle\operatorname{Sgn} H_{j}(0), \varphi\right\rangle=2^{j+1} j{\underset{\lambda=-j-1}{\lambda=s}}_{\operatorname{Res}}\left\langle\operatorname{sgn} x_{n} Q(x, x)_{-}^{\lambda}, \varphi\right\rangle
$$

and in place of (3.17) we have

$$
\begin{aligned}
\left\langle\operatorname{sgn} x_{n} Q(x, x)_{-}^{\lambda}, \hat{\varphi}\right\rangle= & -i \pi^{-2 \lambda-1-n / 2} \Gamma(\lambda+1) \\
& \cdot \Gamma\left(\lambda+\frac{n}{2}\right) \sin \pi\left(\lambda+\frac{p-1}{2}\right)\left\langle\operatorname{sgn} x_{n} Q(x, x)_{-}^{\lambda}, \varphi\right\rangle .
\end{aligned}
$$

\section{REFERENCES}

1. I. M. Gelfand and G. E. Shilov, Generalized functions. Vol. I, Academic Press, New York, 1964.

2. E. M. Stein and G. Weiss, Introduction to Fourier analysis on Euclidean spaces, Princeton Univ. Press, Princeton, N. J., 1971.

3. R. S. Strichartz, Fourier transforms and non-compact rotation groups, Indiana Univ. Math. J. 24 (1974), 499-526; Errata 30 (1981), 479-480.

4a. M. Vergne, A Plancherel formula without group representations, Lecture, O.A.G.R. Conference, Bucharest, Roumania, 1980.

4b. , A Poisson-Plancherel formula for semi-simple Lie groups, preprint.

Department of Mathematics, Cornell University, IthaCa, New York 14853 